\title{
Consenso sobre o tratamento da dependência de nicotina
}

\section{Consensus on the treatment of nicotine dependence}

Ana Cecilia P R Marques (SP), Angelo Campana (RS), Analice de Paula Gigliotti (RJ), Maria Teresa C Lourenço (SP), Montezuma Pimenta Ferreira (SP) e Ronaldo Laranjeira (SP)

Departamento de Dependência Química da Associação Brasileira de Psiquiatria

\section{Apresentação}

Os protocolos, as diretrizes, os guidelines ou guias de conduta ou consensos surgiram graças ao EvidenceBased Medicine Working Group, dos Estados Unidos, que elaborou as bases científicas desses métodos. $\mathrm{O}$ objetivo é a elaboração de práticas recomendadas ao médico em relação a diversos temas. É a compilação de um conjunto de orientações a ser seguidas, preservando a autonomia do médico. Para elaborar um consenso formal, isto é, aquele em que se define previamente o tema, reúnem-se especialistas com a tarefa de realizar a revisão sistemática da literatura para organizar o conhecimento científico. As associações médicas têm desempenhado um papel fundamental em auxiliar seus afiliados nessa tarefa. A Associação Médica Brasileira (AMB) tem considerado a criação de consensos como uma de suas mais importantes metas.

O Departamento de Dependência Química da Associação Brasileira de Psiquiatria optou por começar seus trabalhos com a realização do Consenso sobre a sindrome de abstinência do álcool, publicado em junho de 2000 por esta revista. Este ano desenvolveu-se o Consenso sobre a dependência de nicotina. Um pouco mais experientes, mas não menos cuidadosos, com a mesma metodologia utilizada na elaboração do consenso anterior, acreditamos ter cumprido mais essa tarefa, que agora vemos também publicada na Revista Brasileira de Psiquiatria. A divulgação, a discussão e a atualização continuada dessas diretrizes devem ser consideradas prioridades dessa associação e desse departamento, que, diante de temas tão complexos, ainda compartilhará muito trabalho pela frente.

AEquipe

Recebido em 16/5/2001. Revisado em 20/6/2001 e 8/10/2001. Aceito em 9/10/2001. Fonte de financiamento: Pharmacia \& Upjohn Ltda.

Conflito de interesses inexistente.

\section{Resumo}

Têm sido atribuídas à dependência de nicotina $20 \%$ das mortes nos EUA. Estudos têm mostrado que $30 \%$ a $50 \%$ das pessoas que começam a fumar escalam para um uso problemático. Nos últimos 20 anos, a educação e a persuasão não foram suficientes para promover uma mudança política, cultural e social relacionada ao comportamento de fumar. As intervenções para interromper o uso de tabaco ainda não estão integradas às rotinas dos serviços de saúde no mundo. A falta de estratégias de integração, de tempo disponível para acoplar ações assistenciais mais específicas e mesmo a percepção dos profissionais de saúde de que os tratamentos para a dependência de nicotina são pouco efetivos são algumas das barreiras apontadas. Assim, elaborar um consenso sobre a dependência de nicotina teve como objetivos:

- levantar dados epidemiológicos relevantes relacionados ao uso do tabaco no mundo e no Brasil;

- revisar as ações gerais e centrais da nicotina;

- elaborar um protocolo de triagem mínimo para serviços de atenção primária à saúde;

- recomendar diretrizes básicas de avaliação, diagnóstico e tratamento para todos os níveis de atenção à saúde em relação à dependência de nicotina;

- fornecer sugestões para a abordagem de grupos especiais de pacientes: adolescentes, gestantes, idosos, pacientes em regime de internação, obesos e pacientes com comorbidades psiquiátricas, cardiovasculares e respiratórias.

\section{Descritores}

Dependência de nicotina. Tratamento.

\begin{abstract}
Twenty percent of mortality rates in the US have been attributed to nicotine dependence. Thirty to fifty percent of nicotine dependent patients will reach a stage of problematic use in a short-term follow-up. Over the last 20 years, educational programs and persuasive techniques aimed to the general population were not able to have a significant impact on political, cultural and social beliefs to change smoking behavior. Treatment programs to stop smoking are not yet integrated in health serv-
\end{abstract}


ices routines worldwide. The lack of integration among different levels of prevention programs; general practitioners' perception that treatment outcome is not effectual, and time unavailability to further assess the problem are considered the main barriers to achieve successful treatment results. Thus, the objectives of creating a consensus about nicotine dependence were the following:

- gather major epidemiological data about tobacco use worldwide and in Brazil;

- review general and central actions of nicotine in the body;

- create a protocol for screening primary health service;

- set guidelines for assessment, diagnosis and treatment to be applied in the different levels of public health services;

- give recommendations about approaches for specific groups: adolescents, pregnant women; elderly people; obese, hospitalized patients, and psychiatric patients, and for patients with heart and respiratory conditions.

\section{Keywords Nicotine dependence. Treatment.}

\section{Diretrizes básicas sobre a dependência de nicotina}

\section{Introdução}

A difusão do tabaco no começo do século XX fez com que seu consumo aumentasse durante a $2^{\mathrm{a}}$ Grande Guerra Mundial, atingindo o auge nas décadas de 50 e 60 . Declinou discretamente em alguns países desde 1970, mas os problemas associados a esse consumo têm estimulado mais pesquisas, pois os dados são alarmantes. Existem mais de 1 bilhão de fumantes no mundo, e a metade deles, se permanecer fumando, morrerá prematuramente. ${ }^{1,2}$

Em 1990, 20\% das mortes nos EUA foram atribuídas ao tabagismo. ${ }^{3}$ Entre as 25 doenças relacionadas ao hábito de fumar, são causas de morte em homens e em mulheres, em ordem de incidência: doenças cardiovasculares (43\%); câncer (36\%); doenças respiratórias $(20 \%)$; e outras $(1 \%){ }^{3-5}$

Já em 1988, um importante relatório norte-americano concluiu que: (1) o cigarro e outras formas de uso do tabaco geram dependência; (2) a nicotina é a droga presente no tabaco que causa essa dependência; (3) os processos farmacológicos e comportamentais que determinam a dependência de nicotina são similares àqueles que determinam a dependência de outras drogas como a heroína e a cocaína ${ }^{6}$

A prevalência da dependência de nicotina na população adulta norte-americana diminuiu, desde 1965 , de $42 \%$ para $25 \%$, e, desde então, esse número não se modificou. ${ }^{7} \mathrm{Um}$ dos aspectos relevantes que explicam parte desse fenômeno é que apenas $2,5 \%$ de todos os fumantes alcançam a abstinência a cada ano. ${ }^{8,9}$ No Brasil, a prevalência observada em um estudo recente na população adulta de São Paulo é semelhante à norte-americana: em torno de $24 \% .^{10}$

Estudos têm mostrado que $30 \%$ a $50 \%$ das pessoas que começam a fumar escalam para um uso problemático. ${ }^{11,12} \mathrm{O}$ Serviço Nacional de Saúde dos Estados Unidos mostrou que entre os $70 \%$ dos adolescentes que começam a fumar, $25 \%$ passam rapidamente a fumar todos os dias. ${ }^{13} \mathrm{O}$ uso das demais drogas nessa população declina com a idade, mas isto não acontece com o tabaco. A idade média de início de consumo é 13-14 anos nos EUA e no Brasil, mas a vulnerabilida-
Projeções para 2000-2024 e 2025-2049

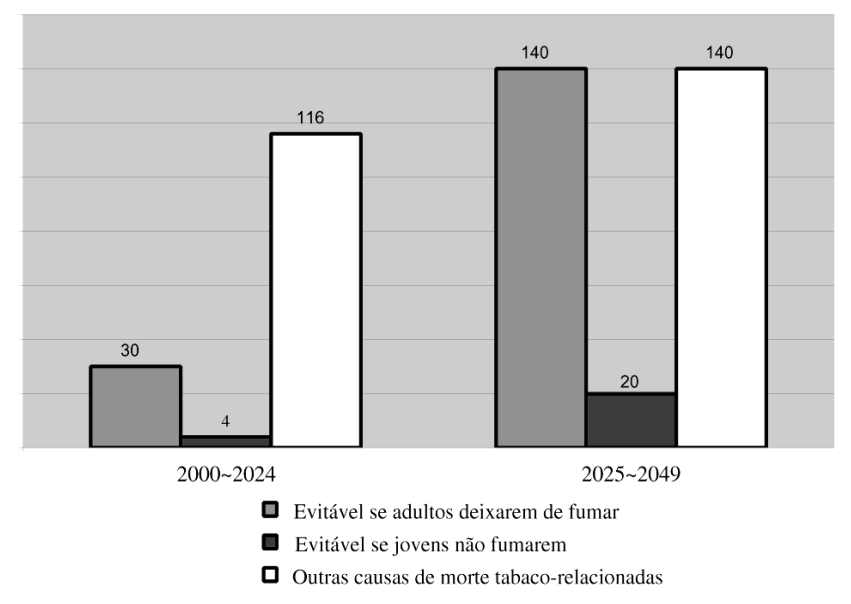

Figura 1 - Mortes prematuras pelo uso do tabaco.

de para dependência não está relacionada apenas à idade..$^{13,14}$

A expectativa de vida de um indivíduo que fuma muito é $25 \%$ menor que a de um não fumante. ${ }^{15}$ Nos últimos 20 anos, a educação e a persuasão não foram suficientes para promover uma mudança política, cultural e social relacionada ao comportamento de fumar. Investimentos continuados e mais consistentes voltados para a prevenção e o tratamento são necessários em função das projeções sobre a mortalidade no período de 2000 a $2049^{7,16}$ (Figura 1).

As intervenções para interromper o uso de tabaco ainda não estão integradas às rotinas dos serviços de saúde no mundo. A falta de estratégias de integração, de tempo disponível para acoplar ações assistenciais mais específicas e mesmo a percepção dos profissionais de saúde de que os tratamentos para a dependência de nicotina são pouco efetivos são algumas das barreiras apontadas. ${ }^{17,18}$ A efetividade dos tratamentos ainda é baixa, mas demonstrou-se que, com aumento dos recursos durante o tratamento, melhora-se o sucesso das intervenções. ${ }^{19}$

Assim, diante de dados tão alarmantes em relação à mortalidade, à morbidade e ao alto custo socioeconômico com a saúde determinados pelo uso de tabaco, além das dificuldades 
na implementação do tratamento, organizou-se um conjunto de recomendações básicas para o tratamento da dependência de nicotina, com o objetivo de sistematizar as práticas clínicas, psicológicas e sociais.

O levantamento da literatura internacional, por meio das publicações da Associação Psiquiátrica Norte-Americana, do Departamento de Saúde dos Estados Unidos e do Departamento de Saúde do Reino Unido (United States Department of Health and Human Services; United Kingdon Institute of National Health and The Tobacco Advisory Group of The Royal College of Physicians of London), forneceu dados para a elaboração deste consenso, cujos objetivos foram:

- levantar dados epidemiológicos relevantes relacionados ao uso do tabaco no mundo e no Brasil;

- revisar as ações gerais e centrais da nicotina;

- elaborar um protocolo de triagem mínimo para serviços de atenção primária à saúde;

- recomendar diretrizes básicas de avaliação, diagnóstico e tratamento para todos os níveis de atenção à saúde em relação à dependência de nicotina;

- fornecer sugestões para a abordagem de grupos especiais de pacientes: adolescentes, gestantes, idosos, pacientes em regime de internação, obesos, pacientes com comorbidades psiquiátricas, cardiovasculares e respiratórias.

\section{Aspectos gerais da ação farmacológica da nicotina}

A queima de um cigarro produz monóxido de carbono e dezenas de outros produtos tóxicos responsáveis pela alteração da oxigenação dos tecidos. Libera nicotina, a substância responsável pela dependência do tabaco, uma amina terciária volátil capaz de estimular, deprimir ou perturbar o sistema nervoso central e todo o organismo, dependendo da dose e da freqüência com que é utilizada. ${ }^{20}$ Cada cigarro contém $7-9 \mathrm{mg}$ de nicotina, dos quais se estima que pouco mais de $1 \mathrm{mg}$ seja absorvido pelo fumante. A nicotina é rapidamente absorvida pelos pulmões, atingindo o cérebro em dez segundos e sendo distribuída para todos os sistemas..$^{21}$ A meia-vida de eliminação da nicotina é de aproximadamente duas horas. Sua metabolização ocorre principalmente no fígado. Apenas $5 \%$ da nicotina são excretados em sua forma original pelos rins. ${ }^{6}$ Seu metabólito principal é a cotinina, cuja deteç̧ão pode ser sistematizada como um coadjuvante no tratamento da dependência de nicotina, monitorando a abstinência.

A nicotina promove um rápido, mas pequeno aumento do estado de alerta, melhorando a atenção, a concentração e a memória em animais. ${ }^{22}$ Além disso, diminui o apetite. ${ }^{15,23}$

Tragar um cigarro produz um rápido efeito estimulante no sistema nervoso central, semelhante àquele descrito pelos usuários de cocaína/crack. ${ }^{24}$ Esse efeito, em contraposição aos sintomas desagradáveis da falta da substância no cérebro, pode contribuir para a dificuldade na manutenção da abstinência, pois, entre os fumantes que já tentaram parar de usar o tabaco, cinco a sete tentativas são necessárias. ${ }^{15}$

A sensação de relaxamento e calma descrita pela maioria dos usuários tem sido atribuída à inibição de sintomas desagradáveis da síndrome de abstinência em vários estudos. ${ }^{25,26}$
As conseqüências do uso do tabaco incluem efeitos destrutivos em vários tecidos, produzindo desde doenças pulmonares simples até alterações celulares que predispõem ao câncer, assim como alterações cardíacas e vasculares.

\section{A neurofarmacologia da nicotina}

As ações da nicotina no sistema nervoso central são mediadas por receptores nicotínicos. ${ }^{27,28}$ A maioria desses receptores é iônica, está distribuída por todo o cérebro e coluna vertebral. Os receptores nicotínicos periféricos estão em gânglios autonômicos, na supra-renal, nos nervos sensitivos e na musculatura esquelética. ${ }^{29}$

As pesquisas em animais mostram que a nicotina induz tolerância e dependência a partir de sua ação no sistema mesolímbico dopaminérgico. ${ }^{30,31}$

A partir de múltiplos sítios de ação, a nicotina produz vários efeitos, preponderantemente excitatórios ${ }^{32}$ (Figura 2). A administração aguda de nicotina em ratos pode aumentar a liberação de dopamina no sistema límbico e na via nigroestriatal. ${ }^{33,34} \mathrm{~A}$ nicotina diminui o metabolismo da glicose no córtex, refletindo na modulação dopaminérgica do núcleo acumbens, causando uma diminuição da atividade talâmica. ${ }^{35}$

A nicotina estimula a liberação de noradrenalina em algumas áreas, como as vias noradrenérgicas que emergem do locus ceruleus, passam pelo hipocampo e chegam ao córtex e são responsáveis pela vigília e pelo comportamento de busca. ${ }^{36,37}$

Estudos mais recentes identificaram que vias serotoninérgicas

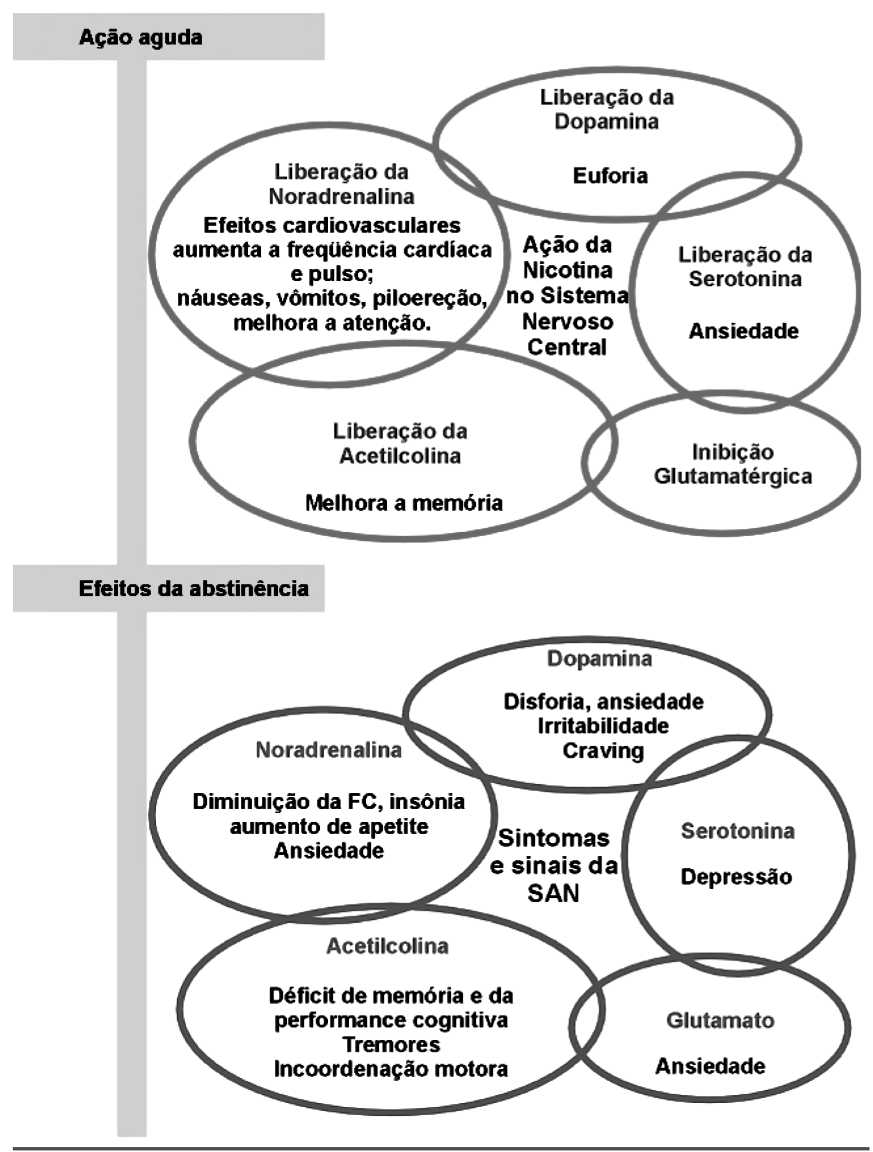

Figura 2 - Bases biológicas da nicotina no sistema nervoso central. 
que emergem do núcleo da rafe interagem com o sistema dopaminérgico e são responsáveis pelos efeitos reforçadores da cocaína. ${ }^{38}$ Receptores nicotínicos foram encontrados nela, onde a nicotina promoveria a liberação da serotonina, além de diminuir seu turnover.$^{39}$ A nicotina diminui a concentração de serotonina em regiões do hipocampo, o que, cronicamente, reduz a resposta de adaptação ao estresse ambiental. ${ }^{40}$

Em relação aos aminoácidos excitatórios, a nicotina agiria inibindo a liberação do glutamato. Já em relação à acetilcolina, a nicotina liberaria esse neurotransmissor no hipocampo. ${ }^{41}$

Estudos eletroquímicos em animais mostraram que os efeitos comportamentais da nicotina advêm do efeito direto no sistema nervoso central..$^{42}$ Seu uso produz tolerância aguda e crônica nesse sistema. ${ }^{43} \mathrm{Nem}$ todas suas ações são reforçadoras, mas a auto-administração de nicotina intravenosa foi diminuída por seu antagonista: o chlorisondamine. ${ }^{44}$

Concluindo, o uso freqüente de tabaco leva ao desenvolvimento de tolerância e dependência. ${ }^{45}$ Como outras dependências, a da nicotina é um transtorno progressivo, crônico e recorrente, mediada pela ação da substância em receptores centrais e periféricos. O processo da dependência envolve vários fatores: ambientais, biológicos, psicológicos. Fatores não-farmacológicos são importantes para a determinação dos problemas relacionados ao uso de tabaco e sua prevalência, mas os fatores farmacológicos são aqueles que definem a tolerância e a dependência pela substância. ${ }^{46}$

Alguns fatores inerentes ao indivíduo, ainda pouco conhecidos, podem contribuir para o início do consumo de cigarros. Entre eles, os genético-hereditários, que poderiam estar relacionados à modulação do humor pela nicotina. ${ }^{47,48}$ Existem também outros aspectos relacionados ao uso do tabaco na adolescência, como a utilização do tabaco pelos pais, pelos colegas mais velhos e a influência da mídia, considerados fatores preditores de consumo. ${ }^{13,49}$

Estima-se que $60 \%$ daqueles que venham a fumar por mais de seis semanas continuarão fumando por mais 30 anos. Embora o primeiro uso do cigarro seja tipicamente marcado por efeitos desagradáveis, como dor de cabeça, tonturas, nervosismo, insônia, tosse e náusea, esses efeitos diminuem rapidamente. ${ }^{50}$ Isto possibilita novas tentativas até que se desenvolva tolerância à droga, estabelecendo um padrão típico de consumo diário. Num período que pode ser de apenas alguns meses, alguns fumantes já começam a apresentar os primeiros sintomas de uma síndrome de abstinência. ${ }^{6}$

A decisão de experimentar uma droga se relaciona a uma expectativa de efeitos positivos, mas a explicação para esse fenômeno ainda não está claramente evidenciada ${ }^{52}\left(\right.$ Heishman $^{51}{ }^{5}$ 1994). No caso da nicotina, os efeitos estimulantes são qualitativamente semelhantes aos da cocaína e da anfetamina, mas os fumantes também procuram na nicotina o alívio da ansiedade e a diminuição da reatividade a estímulos que causam irritação (Figura 2) ${ }^{6,53}$

Nos EUA, $90 \%$ dos fumantes usam mais que cinco cigarros ao dia. A maioria dos fumantes que já experimentaram reduzir o uso descreve vários sintomas de abstinência. ${ }^{54} \mathrm{~A}$ síndrome de abstinência se instala se o consumo for reduzido em apenas $50 \%{ }^{55}$ Parece que quanto maior o consumo, maior a gravidade da síndrome de abstinência. ${ }^{6}$ Os sintomas e a magnitude da síndrome de abstinência podem persistir por meses e, dependendo de sua gravidade, são pouco tolerados. ${ }^{40,56} \mathrm{Em}$ função desse desconforto, é difícil para os usuários manter a abstinência..$^{57,58}$

A síndrome de abstinência é mediada pela noradrenalina, inicia-se oito horas após o último cigarro, atinge o auge no terceiro dia e inclui craving ou "fissura", ansiedade, irritabilidade, sonolência diurna e insônia, apetite aumentado para doces, desempenho cognitivo (concentração e atenção), batimentos cardíacos e pressão arterial diminuídos e tosse (Tabela 1). ${ }^{59}$

Tabela 1 - Síndrome de abstinência de nicotina.

Síntese dos sintomas e sinais da síndrome de abstinência de nicotina

Psicológicos:

Humor disfórico ou deprimido

Insônia e sonolência diurna

Irritabilidade, frustração ou raiva

Ansiedade

Dificuldade para concentrar-se e para manter a atenção

Inquietação

"Fissura" ou craving

Biológicos:

Freqüência cardiaca diminuída

Pressão arterial diminuída

Aumento do apetite

Ganho de peso

Incoordenação motora e tremores

Sociais:

Relacionamento social instável em conseqüência do estado ansioso

\section{Diagnóstico}

O consumo de tabaco geralmente começa na adolescência. Quanto mais precoce o início, maiores serão a gravidade da dependência e os problemas a ela associados. ${ }^{60}$ Além disso, a maioria dos fumantes que alcançam a abstinência sozinhos recai em poucos dias. ${ }^{61}$

A relutância do fumante em procurar ajuda e a falta de serviços dotados de profissionais treinados para detectar o paciente de risco são barreiras na procura de tratamento. ${ }^{62}$

Todos os indivíduos que chegam aos serviços de saúde devem ser investigados quanto ao hábito de fumar. Isto pode ser feito a partir de um protocolo simples com apenas algumas perguntas (Anexo 1). Se fumam, devem ser aconselhados a interromper o uso de tabaco. ${ }^{63}$ Caso não seja possível aconselhar adequadamente, é melhor encaminhar o fumante para um serviço especializado.$^{19}$ Neste, duas perguntas são fundamentais para a triagem e para o início do diagnóstico do indivíduo que fuma: ${ }^{64}$

- qual o consumo diário de tabaco;

- se o fumante acredita ter problemas associados a esse consumo.

Essa avaliação mínima também pode ser aplicada por qualquer profissional, em qualquer nível de atenção à saúde, pois é simples, objetiva e breve. A essas perguntas, segue-se um protocolo de aconselhamento com dois objetivos: orientar aqueles que desejem parar de fumar ou motivar aqueles que não quiserem largar o cigarro. Essa intervenção tem se mostrado efetiva (Figura 3) ${ }^{7,65}$ 


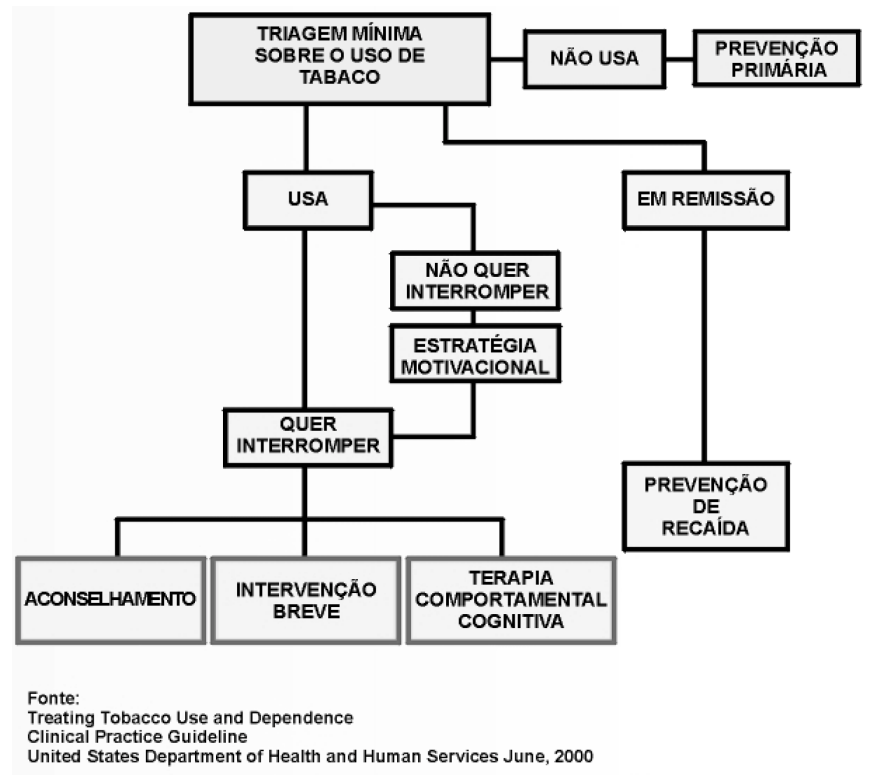

Figura 3 - Avaliação inicial do fumante em qualquer nível de atenção à saúde.

Questionários, inventários e escalas desenvolvidos para elaborar o diagnóstico e melhor avaliar a gravidade do consumo de tabaco podem ser introduzidos para fundamentar a intervenção. A Classificação Internacional das Doenças (CID10) da Organização Mundial da Saúde ${ }^{66}$ e a quarta edição do Manual de Diagnóstico e Estatístico (DSM-IV) da Associação Psiquiátrica Americana ${ }^{67}$ são utilizados para avaliação diagnóstica do uso do tabaco. Recomenda-se a utilização da CID-10 (Tabela 2).

O "Questionário de Tolerância de Fagerström para Dependência à Nicotina" (Anexo 2) $;^{40}$ o Tobacco Dependence
Screener $^{68}$ e o The Heaviness of Smoking Item $^{69}$ avaliam sua gravidade e são mais utilizados em serviços especializados com maior complexidade de recursos.

\section{Tratamento}

Existem vários modelos de tratamento propostos, e a escolha do mais adequado ao paciente depende de uma boa avaliação inicial, pela qual fatores extrínsecos (do modelo disponível, das condições socioeconômicas) e intrínsecos (da motivação do paciente e do diagnóstico) devem ser levados em consideração. ${ }^{23}$

Em todos os tratamentos, a abstinência é a meta mais importante e a mais difícil de ser mantida. A abordagem de um dos sintomas mais proeminentes da síndrome de abstinência, o craving ou "fissura", deve ser cuidadosamente considerada, já que este é $o$ maior obstáculo para parar de fumar. ${ }^{57,70,71}$ A maioria dos fumantes que tentam se manter abstinentes recai em poucos dias. ${ }^{72}$ Com algum nível de cuidado, o período de abstinência aumenta para uma semana ou mais (Tabela 3 ). ${ }^{73}$

Segundo pesquisadores americanos e ingleses, o tratamento pode ser definido a partir do consumo de cigarros (gravidade e problemas associados) e da modalidade de intervenção disponível. Os métodos de tratamento preconizados como primeira linha são a terapia de reposição de nicotina, a utilização de bupropiona e a terapia comportamental breve em grupo ou individual.

Os grupos de auto-ajuda e outros medicamentos são considerados de segunda linha e podem ser coadjuvantes efetivos..$^{74} \mathrm{~A}$ associação de recursos melhora sua efetividade. ${ }^{75}$

As sessões de aconselhamento podem ser mínimas (3 minutos), de baixa intensidade (de 3 a 10 minutos) e intensivas (de 10 a 30 minutos). ${ }^{68}$ Quanto mais intensiva é a intervenção, melhores serão os resultados em longo prazo. ${ }^{76}$

Tabela 2 - Classificação de Transtornos Mentais e de Comportamento (CID-10, WHO, 1992).

\begin{tabular}{|c|c|}
\hline \multicolumn{2}{|r|}{ Diretrizes diagnósticas do CID-10 para dependência de nicotina F15: somente se três ou mais requisitos estão presentes durante o último ano: } \\
\hline $\begin{array}{l}\text { a. } \\
\text { b. } \\
\text { c. } \\
\text { d. }\end{array}$ & $\begin{array}{l}\text { um forte desejo ou senso de compulsão para consumir a substância; } \\
\text { dificuldade em controlar o comportamento de consumir a substância em termos de seu início, término ou níveis de consumo; } \\
\text { um estado de abstinência fisiológico quando o uso da substância cessou ou foi reduzido, como evidenciado por: síndrome de abstinência } \\
\text { característica para a substância ou o uso da mesma substância (ou de uma substância intimamente relacionada) com a intenção de aliviar ou evitar } \\
\text { sintomas de abstinência; } \\
\text { evidência de tolerância, de tal forma que doses crescentes da substância psicoativa são requeridas para alcançar efeitos originalmente produzidos } \\
\text { por doses mais baixas (exemplos claros disto são encontrados em indivíduos dependentes de álcool e opiáceos, que podem tomar doses diárias } \\
\text { suficientes para matar ou incapacitar usuários não tolerantes); } \\
\text { abandono progressivo de prazeres ou interesses alternativos em favor de uso da substância psicoativa, aumento da quantidade de tempo necessá } \\
\text { rio para obter ou tomar a substância ou para se recuperar de seus efeitos; } \\
\text { persistência do uso da substância, a despeito de evidência clara de consequêencias manifestamente nocivas. Deve-se fazer esforços para } \\
\text { determinar se o usuário estava realmente (ou se poderia esperar que estivesse) consciente da natureza e extensão do dano. }\end{array}$ \\
\hline \multicolumn{2}{|r|}{ Diretrizes diagnósticas do CID-10 para estado de abstinência de nicotina F15: } \\
\hline $\begin{array}{l}\text { - un } \\
\text { usu } \\
\text { sub } \\
\text { Per } \\
\text { Per }\end{array}$ & $\begin{array}{l}\text { onjunto de sintomas de agrupamentos e gravidade variáveis, ocorrendo em abstinência absoluta ou relativa de nicotina, após uso repetido e } \\
\text { eente prolongado e/ou de altas doses daquela substância. O início e curso do estado de abstinência são limitados no tempo e relacionados ao tipo de } \\
\text { ncia e a dose que vinha sendo utilizada imediatamente antes da abstinência. } \\
\text { bações psicológicas: } \\
\text { Humor disfórico ou deprimido } \\
\text { Insônia } \\
\text { Irritabilidade, frustração ou raiva } \\
\text { Ansiedade } \\
\text { Dificuldade para concentrar-se } \\
\text { Inquietação } \\
\text { bações físicas: } \\
\text { Freqüência cardíaca diminuída } \\
\text { Aumento do apetite ou ganho de peso }\end{array}$ \\
\hline
\end{tabular}


Tabela 3 - Aspectos relevantes da síndrome de abstinência de nicotina: tempo de duração dos sintomas e sinais; sua relação com a terapia de substituição, com a recaída e a influência ou não de algum tipo de tratamento.

\begin{tabular}{|c|c|c|c|c|c|}
\hline \multicolumn{6}{|c|}{ Principais sinais da síndrome de abstinência de nicotina } \\
\hline Sintomas & Duração & $\begin{array}{l}\text { Reduzido } \\
\text { pela TRN }\end{array}$ & $\begin{array}{l}\text { Fatores de } \\
\text { recaída }\end{array}$ & $\begin{array}{c}\text { Incidência } \\
\text { Cessam sem } \\
\text { Tratamento (\%) }\end{array}$ & $\begin{array}{c}\text { Pacientes } \\
(\%)\end{array}$ \\
\hline Irritabilidade/agressividade & $<4$ semanas & Sim & Não & 38 & 80 \\
\hline Depressão & $<4$ semanas & Sim & Sim & 31 & 69 \\
\hline Ansiedade & $<2$ semanas & Sim & Não & 49 & 87 \\
\hline Insônia & $<2$ semanas & Sim & Não & 46 & 71 \\
\hline Concentração pobre & $<1$ semana & Sim & Não & 43 & 73 \\
\hline Aumento do apetite & $>10$ semanas & Sim & Não & 53 & 67 \\
\hline "Fissura" & $>2$ semanas & Sim & Sim & 37 & 62 \\
\hline Despertares nortunos & $<1$ semana & $\mathrm{d}$ & Não & 39 & 24 \\
\hline Diminuição da freqüência cardíaca & $>10$ semanas & Sim & $\mathrm{d}$ & 61 & 79 \\
\hline Diminuição da adrenalina & $<2$ semanas & $\mathrm{d}$ & $d$ & $d$ & $\mathrm{~d}$ \\
\hline Diminuição do cortisol & d & $d$ & $d$ & d & $\mathrm{d}$ \\
\hline
\end{tabular}

Diminuição do cortisol

$d=$ desconhecido;

TRN = Terapia de Reposição de Nicotina;

Fonte: Gross J, Stitzer ML, 1989.

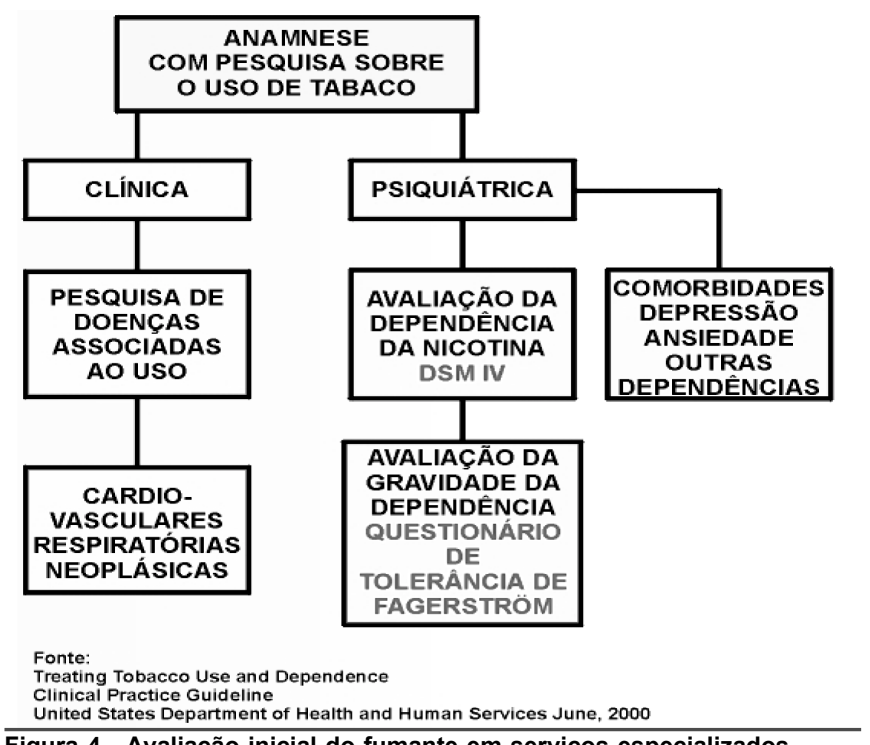

Figura 4 - Avaliação inicial do fumante em serviços especializados.

Nos tratamentos especializados, a avaliação inicial investiga, além da história detalhada do uso de tabaco na vida, comorbidades psiquiátricas mais prevalentes, como transtorno depressivo ou ansioso, outras dependências e déficit de atenção e hiperatividade, entre outros (Figura 4). ${ }^{77-79}$

\section{O aconselhamento}

Freqüentam serviços gerais de saúde $90 \%$ dos fumantes americanos, sendo que $80 \%$ visitam o clínico geral pelo menos uma vez ao ano. ${ }^{80}$ Nesses serviços, um aconselhamento mínimo pode ser aplicado, pois é a forma de intervenção mais utilizada na abordagem individual ou grupal (Anexo 3). Essa intervenção consiste em: (1) perguntar sobre o uso de tabaco; (2) aconselhar sua cessação; (3) investigar sobre o desejo de interromper o uso; (4) oferecer assistência e acompanhamento do processo; e (5) efetuar seu seguimento (Tabela 4).

Caso o fumante não esteja interessado em interromper o uso, estratégias motivacionais devem ser implementadas. São elas: informar sobre aspectos individuais relevantes para cessação; relatar sobre os riscos do tabagismo (agudo, crônicos e ambi-
Tabela 4 - Etapas do aconselhamento.

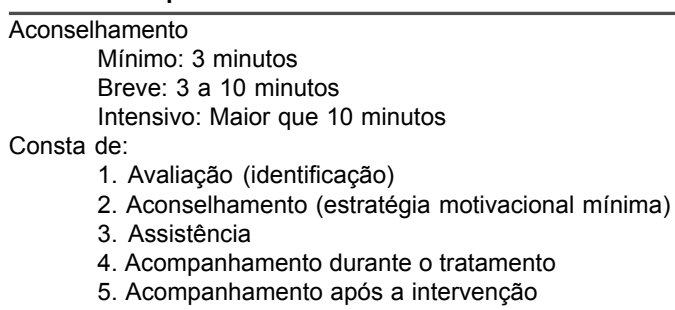

entais); descrever as recompensas decorrentes da cessação; investigar as barreiras na procura do tratamento; e repetir os tópicos relevantes da avaliação inicial (Tabela 5).

Para aqueles que desejem interromper o uso, uma abordagem mais intensiva e um tratamento mais estruturado como a intervenção breve podem ser prontamente indicados. ${ }^{81,82} \mathrm{Se}$ essa ação falhar, o paciente deve ser encaminhado para o especialista. Materiais didáticos podem ser também utilizados.

\section{A intervenção breve}

A intervenção breve é um modelo de tratamento mais estruturado, replicável em qualquer nível de atenção à saúde, pois seu formato é simples e, portanto, de fácil treinamento ${ }^{83} \mathrm{O}$ referencial teórico mais recomendado para essa intervenção é a terapia comportamental, e a abordagem mais utilizada é a grupal. ${ }^{84,85}$ Essa modalidade pode envolver até 25 pacientes, sendo considerada de baixo custo. ${ }^{86}$

A entrevista inicial é individual e direcionada para história de uso do tabaco na vida, para gravidade do consumo, para problemas associados, para aspectos relacionados a tratamentos anteriores (quantas vezes tentou interromper o uso) e para avaliação do grau de motivação para o tratamento.

A fase de aquisição da intervenção em grupo é composta de quatro ou cinco sessões. A sessão tem a duração de uma hora, e sua freqüência depende do contrato. Na primeira sessão do grupo, faz-se a apresentação e fornecem-se algumas informações sobre cada um dos pacientes. Discute-se também a utilização da terapia de reposição da nicotina como parte da intervenção, se esta tiver sido indicada. A seguir, é feito o aconselhamento sobre a importância de interromper o uso e sobre os riscos associados à saúde, de uma forma clara, firme e 


\begin{tabular}{|c|c|}
\hline \multicolumn{2}{|l|}{$\begin{array}{l}\text { Estratégias motivacionais } \\
\text { Recompensas ou vantagens }\end{array}$} \\
\hline $\begin{array}{l}\text { Melhora a saúde } \\
\text { Paladar e olfato estarão mais aguçados } \\
\text { Fará economia }\end{array}$ & $\begin{array}{l}\text { Agudos: dificuldade respiratória, asma, impotência, infertilidade, } \\
\text { alterações no feto. }\end{array}$ \\
\hline $\begin{array}{l}\text { A casa, as roupas, o carro e o seu hálito terão melhor odor } \\
\text { Você poderá se despreocupar sobre parar de fumar } \\
\text { Você será um bom exemplo para as crianças } \\
\text { Seus filhos terão mais saúde }\end{array}$ & $\begin{array}{l}\text { Crônicos: acidentes vasculares hemorrágicos e infarto, câncer e laringe, } \\
\text { boca, faringe, esôfago, pâncreas, baço. } \\
\text { Bronquite crônica e enfisema. }\end{array}$ \\
\hline $\begin{array}{l}\text { Você não mais se preocupará em expor os outros ao tabaco } \\
\text { Você se sentirá melhor fisicamente } \\
\text { Seu desempenho nas atividades físicas melhorará } \\
\text { Você reduzirá o envelhecimento de sua pele, diminuindo as rugas }\end{array}$ & $\begin{array}{l}\text { Ambientais: aumento de câncer nos cônjuges, taxas altas de filhos que fumarão, } \\
\text { que nascerão com baixo peso, asma, doenças do ouvido médio e respiratórias. }\end{array}$ \\
\hline \multicolumn{2}{|l|}{ Tabela 6 - Intervenção breve } \\
\hline \multicolumn{2}{|l|}{ Intervenção breve } \\
\hline \multicolumn{2}{|c|}{ 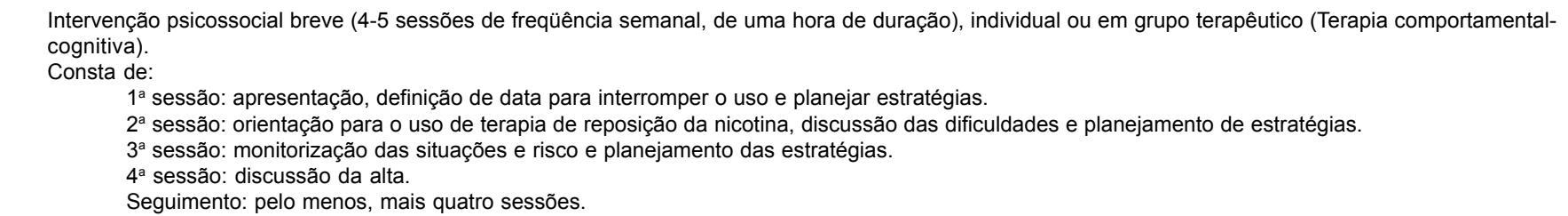 } \\
\hline
\end{tabular}

individualizada. Nessa sessão, determina-se a data de interromper o uso e a proposta contratual de assistência.

Na segunda sessão, pode ser discutida a terapia de substituição com a nicotina, oferecendo aos pacientes todas as explicações necessárias para seu uso, dependendo das dificuldades de cada um. Nas demais sessões, as situações de risco e de proteção são discutidas com o objetivo de traçar estratégias de manutenção da abstinência. Na quarta sessão, os pacientes são orientados quanto à fase de seguimento, acompanhamento após a alta. Esse período pode ser de até mais quatro sessões. A rede social ou familiar, ou ambas, podem ser inserida no tratamento (Tabela 6).

A terapia comportamental-cognitiva também pode ser utilizada, pois a base teórica é a mesma: a teoria do aprendizado social de Bandura, ${ }^{87}$ segundo a qual qualquer uso de drogas é um comportamento aprendido, desencadeado e mantido por eventos e emoções específicos, possível de ser modificado. Esse tratamento também é breve, focal, estruturado em três níveis: a modificação do comportamento de uso e dos pensamentos automáticos disfuncionais, a resolução dos problemas associados e o reajuste social e ambiental. O período de duração da intervenção é mais longo, podendo alcançar de 12 a 16 sessões.

\section{Farmacoterapia}

O único tratamento farmacológico considerado de primeira linha licenciado na Inglaterra é a terapia de substituição da nicotina, que tem como objetivo aliviar os sintomas da síndrome de abstinência da substância. ${ }^{88-90}$ Já nos Estados Unidos, além dessa intervenção, o antidepressivo bupropiona foi aprovado recentemente como medicação de primeira escolha. As demais intervenções farmacológicas como a clonidina e a nortriptilina são consideradas de segunda linha por ambos (Tabela 7).
Tabela 7 - Farmacoterapia e outros métodos de intervenção.

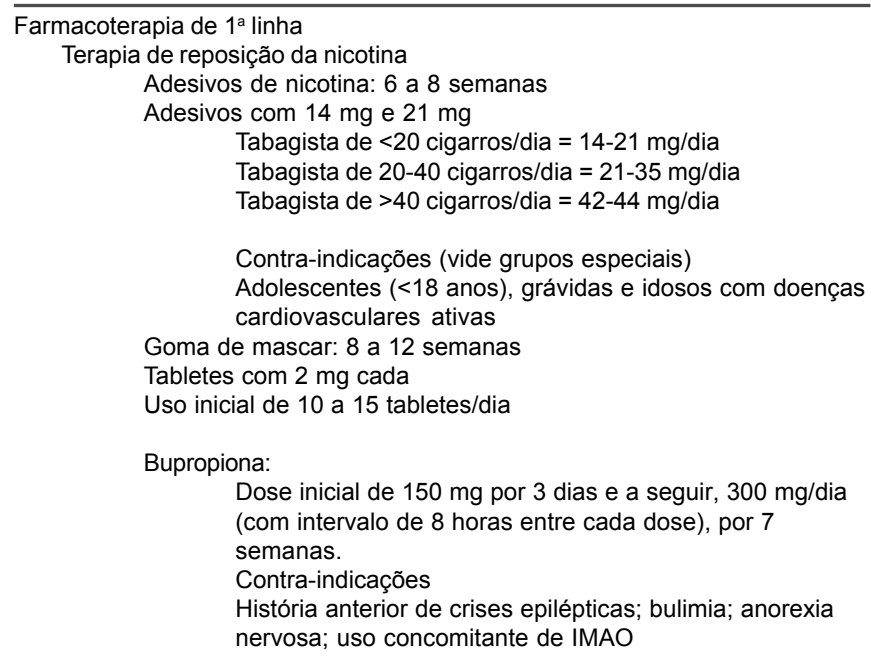

Outros métodos de intervenção

Aconselhamento por telefone

Material didático para a leitura

A associação da terapia de reposição de nicotina com a bupropiona tem resultado em um aumento da efetividade na cessação do uso do tabaco, quando comparada ao uso de bupropiona isoladamente. ${ }^{91}$

\section{A terapia de substituição da nicotina}

Qualquer profissional de saúde treinado pode aplicar a terapia 
de reposição ou substituição com nicotina para pacientes que consomem mais de dez cigarros ao dia. Ela é considerada um método seguro no tratamento da dependência de nicotina, o mais popular e o menos dispendioso. Quando comparada com placebo, é mais efetiva, influenciando também a freqüência das recaídas. ${ }^{21,92,93}$

Esse tratamento pode ser aplicado por quatro formas de apresentação do produto com nicotina: a goma de mascar, o sistema transdérmico, o spray nasal e o vaporizador oral. No Brasil estão disponíveis apenas a goma de mascar e o adesivo de nicotina. $\mathrm{O}$ aconselhamento que acompanha a terapia de reposição com nicotina não é intensivo e, quando administrado em adultos saudáveis, tem produzido resultados positivos. ${ }^{94-97}$

A terapia de reposição alivia os sintomas da síndrome de abstinência. ${ }^{98,99}$ A remissão do uso pode durar seis meses ou mais com esse tratamento. ${ }^{75}$ Quando se associa a terapia de reposição de nicotina a outros recursos terapêuticos (aconselhamento ou outra medicação), a efetividade do tratamento pode aumentar. ${ }^{100,101}$

Para qualquer nível de consumo de tabaco, o uso de $15 \mathrm{mg}$ de nicotina por dia é preconizado como dose inicial. Para fumantes pesados ( 25 cigarros ou mais por dia), a dose inicial pode ser maior. ${ }^{102}$

No Brasil estão disponíveis adesivos com $7 \mathrm{mg}, 14 \mathrm{mg}, 21 \mathrm{mg}$ de nicotina ativa, com utilização pelo prazo médio de oito semanas, trocados diariamente. Essa forma de reposição de nicotina é a mais indicada, pois apresenta menos efeitos colaterais. A redução da dose é progressiva por até um ano.

A goma de mascar encontrada no Brasil contém $2 \mathrm{mg}$ de nicotina ativa por unidade. A média de consumo é de 10 gomas por dia, podendo chegar até 20. Pode produzir irritação na língua e na cavidade oral, mas ainda é o tratamento de menor custo nos países desenvolvidos, quando comparado ao custo de um maço de cigarros consumido por dia. ${ }^{103,104}$ Infelizmente, o custo da terapia de reposição de nicotina não é baixo no Brasil.

Existem algumas restrições para a aplicação da terapia de reposição de nicotina, apesar dessa ser considerada muito mais segura do que fumar. Esse método não deve ser indicado para grávidas, para menores de 18 anos e para aqueles pacientes portadores de doenças cardiovasculares instáveis, como infarto do miocárdio recente, angina instável ou determinadas arritmias.

Com a administração de outras substâncias, além da

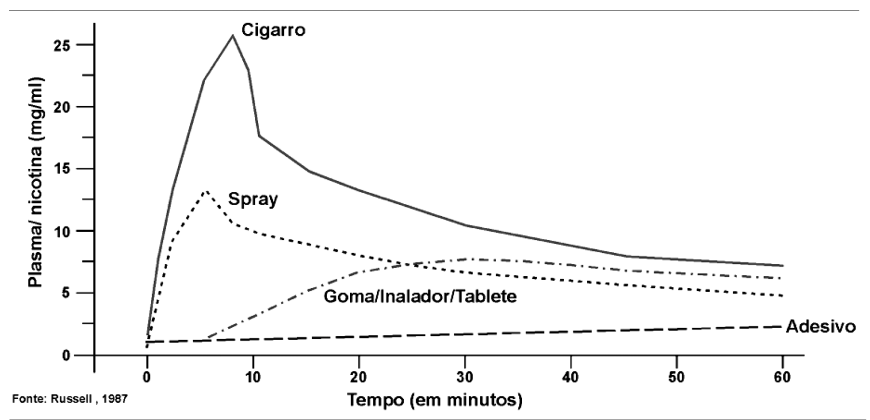

Figura 5 - Diagrama esquemático mostrando a elevação dos níveis de nicotina no sangue venoso após fumar um cigarro e após usar um dos produtos de terapia de substituição da nicotina, seguidos de uma noite de abstinência do uso de cigarros. nicotina, os dependentes de nicotina têm conseguido manter a abstinência em uma porcentagem duas vezes maior que aqueles que utilizaram placebo (Figura 5)., ${ }^{8,105,106}$

A bupropiona é também tratamento de primeira linha nos Estados Unidos, indicada para adultos que consomem 15 cigarros ou mais ao dia. Para fumantes com depressão, a indicação também é mais precisa. ${ }^{107}$ Presume-se que sua ação é mediada pela noradrenalina e pela dopamina, diretamente agindo na diminuição da "fissura" ou craving. ${ }^{93,108-110}$

Inicia-se a bupropiona uma semana antes da cessação. A dose inicial é de $150 \mathrm{mg}$ por dia até o terceiro dia, passando para $300 \mathrm{mg}$ por 7 a 12 semanas. Comparada com placebo, apresentou um período de abstinência duas vezes maior e o ganho do peso menor. ${ }^{108,111}$ As contra-indicações são: condições que impliquem risco de convulsões, como antecedentes das mesmas, traumatismo cranioencefálico, retirada recente de álcool, transtorno bulímico ou anorexia nervosa, uso concomitante de inibidores da monoaminoxidase ou de compostos contendo bupropiona.

Como farmacoterapia de segunda linha, a nortriptilina tem sido comparada à bupropiona em relação a sua efetividade. ${ }^{112}$ Ela inibe a recaptação de noradrenalina e de dopamina no sistema nervoso central, produzindo um efeito antidepressivo. Observou-se que o tratamento prolongado com a droga facilita a função adrenérgica pós-sináptica, resultando em efeito ansiolítico. Os resultados mostraram que ela é efetiva apenas no seguimento de avaliação de curto prazo. ${ }^{112}$

A clonidina tem sido utilizada na dose de $0,1 \mathrm{mg}$ até $0,75 \mathrm{mg}$ ao dia, aliviando consideravelmente os sintomas da síndrome de abstinência, como ansiedade, irritabilidade, cansaço e craving ou "fissura". ${ }^{113}$ Efeitos colaterais como sedação e hipotensão arterial podem dificultar seu uso e levar ao abandono do tratamento. O efeito mais indesejável é a hipotensão ortostática. A suspensão abrupta da clonidina pode produzir crises hipertensivas. ${ }^{114}$

\section{Outros medicamentos ainda sem evidências científicas suficientes}

A mecamilamina é um antagonista da nicotina, que evita o reforço positivo causado por ela. Apresenta muitos efeitos colaterais, como cólicas abdominais, obstipação, boca seca e dores de cabeça. Os estudos demonstram alguma eficácia no início do tratamento, mas os efeitos colaterais levam a índices significativos de abandono no tratamento. ${ }^{115}$

A buspirona, um agonista serotoninérgico com efeito ansiolítico, produz pouca sedação, baixo potencial de abuso e eficácia na redução dos sintomas de abstinência, como a ansiedade, e baixa incidência de efeitos colaterais. ${ }^{116}$

Assim, diversas formas de cuidado com o paciente podem ser inseridas em todos os níveis de atenção à saúde, desde unidades básicas até serviços especializados, hospitais gerais e psiquiátricos, avaliando-se a necessidade e a disponibilidade de recursos de cada local.

\section{Tratamentos compostos}

A associação da psicoterapia e da farmacoterapia tem se 
mostrado a intervenção mais efetiva. ${ }^{19,117-120}$ Materiais didáticos de auto-ajuda, aconselhamento por telefone e estratégias motivacionais ajudam a melhorar a efetividade do tratamento (Anexo 4). ${ }^{121,122}$

A utilização de tratamentos alternativos como a acupuntura e a hipnose tem sido estudada, mas os resultados têm se mostrado pouco efetivos. ${ }^{123}$

\section{Tratamento de grupos especiais}

\section{Comorbidade psiquiátrica}

A dependência de nicotina incide mais freqüentemente em portadores de certos transtornos mentais, como a esquizofrenia e a depressão. ${ }^{113,124,125}$ É provável que esses grupos necessitem de tratamento específico. A bupropiona e a nortriptilina são eficazes para o tratamento da dependência de nicotina na população geral e para o tratamento da depressão, transtorno que incide em torno de duas vezes mais na população de fumantes. ${ }^{48}$

Evidências indicam que entre $15 \%$ a $25 \%$ dos fumantes apresentam dependência de álcool recente. ${ }^{57,126}$ Intervenções para o tratamento do tabagismo não interferem na recuperação da dependência de outras drogas. Portanto, dependentes de nicotina e de outras substâncias devem ser tratados concomitantemente de todas as comorbidades, incluindo psicoterapia e farmacoterapia.

\section{Câncer}

A sobrevida de cinco anos para todos os tipos de câncer gira em torno de $50 \%$. A cessação do tabagismo tem impacto favorável na eficácia do tratamento do câncer (quimioterapia, radioterapia e cirurgia), no menor índice de complicações e desenvolvimento de doenças futuras, assim como na mortalidade relacionada ou não ao câncer. ${ }^{127}$

Deixar de fumar aumenta em várias vezes a chance de não apresentar câncer de pulmão, pâncreas e colo uterino, além de aumentar a sobrevida daqueles que já apresentam a doença. ${ }^{128-130} \mathrm{O}$ tratamento para o tabagismo nesses casos pode ser a reposição de nicotina e a orientação. Apesar desses dados, apenas $1 / 3$ dos pacientes deixa de fumar após o diagnóstico de neoplasia. ${ }^{131}$

\section{Doenças cardiovasculares e respiratórias}

No alcatrão encontram-se elementos carcinogênicos como: hidrocarbonetos, fenóis, benzopirenos, metais pesados, polônio 210, nitrosaminas, DDT, entre outros. ${ }^{132}$ Muitos dos compostos carcinogênicos identificados na fumaça do cigarro não estão presentes na folha do tabaco, mas são formados pela pirólise induzida por meio das altas temperaturas encontradas na ponta do cigarro aceso. Independentemente de outros fatores de risco, fumar pode aumentar o risco de coronariopatias, doença cardíaca, doença pulmonar obstrutiva crônica, câncer de vias aéreas superiores, de trato digestivo alto e de pulmão. ${ }^{133}$

Em pacientes com doenças coronarianas, deixar de fumar reduz em $50 \%$ o risco de morte prematura. ${ }^{128}$ Fumar aumenta em no mínimo duas vezes o risco de acidentes vasculares isquêmicos e aneurismas fatais de aorta. ${ }^{130}$ Observando também os estudos sobre intervenção para cessação do uso de tabaco para esses pacientes, os resultados apontam para uma efetividade baixa, em torno de $30 \%$. $^{134-136}$

O uso do tabaco é a principal causa de doenças respiratórias, e, mesmo motivados, $75 \%$ dos pacientes tratados voltam a fumar em cinco anos. ${ }^{19}$ As taxas de cessação do uso de tabaco de pacientes com doença pulmonar obstrutiva crônica têm se mostrado mais baixas (menor que 10\%) que aquelas obtidas de pacientes com doenças cardiovasculares. ${ }^{137-139}$

A fumaça do cigarro é uma mistura de aproximadamente 5.000 substâncias diferentes, sendo mais de 40 delas reconhecidamente cancerígenas. É composta de uma parte gasosa (que contém monóxido de carbono) e outra particulada (chamada alcatrão), com nicotina e água. Tanto a parte gasosa quanto a particulada induzem o aparecimento de tumores em animais de experimentação. ${ }^{140}$

\section{Idosos}

Existem poucos estudos epidemiológicos longitudinais que abranjam os idosos e o tabagismo. Um levantamento nacional domiciliar inglês, desenvolvido no período de 1972 a 1996, mostrou que este é o único grupo etário que apresentou um tendência consistente à diminuição do fenômeno. ${ }^{141}$

A cessação do uso do tabaco na faixa etária acima dos 65 anos é benéfica em termos de qualidade de vida e longevidade. ${ }^{142}$ A orientação comportamental e a reposição de nicotina podem ser aplicadas.

\section{Jovens}

Para a maioria dos fumantes, a dependência da nicotina se estabelece na adolescência, e, para uma boa parte dos jovens, esse fenômeno ainda ocorre muito antes da permissão legal para se obter o cigarro. ${ }^{143}$ Esse uso em idade tão precoce tem uma implicação importante em longo prazo na saúde individual e pública. ${ }^{144}$

Dos estudos etnográficos e epidemiológicos ingleses, obtevese o dado de que o jovem fumante ou não, assim como o adulto, acredita que fumar relaxa. ${ }^{145}$ Portanto, medidas preventivas difundidas na mídia, cumprimento das leis quanto à venda de cigarros para menores e prevenção primária nas escolas são medidas muito importantes para essa amostra populacional, mas que têm apresentado resultados pouco efetivos. ${ }^{11,146}$ Para essa população, o grupo de orientação comportamental é bem indicado, pois a dificuldade em parar de fumar é a mesma quando se compara esse grupo com os adultos..$^{147,148}$

A intenção de parar de fumar é fator preditivo de cessação em adultos, mas isto não aparece em adolescentes, sendo que o jovem recai mais que o adulto. ${ }^{149}$

\section{Situação socioeconômica}

Grupos com renda mais baixa e menor acesso à educação têm mais dificuldade em abandonar o fumo, assim como acesso mais difícil a programas de tratamento. Estudos de avaliação dos fatores preditores de consumo pesado apontam a baixa renda como um aspecto bastante significativo. ${ }^{150} \mathrm{~A}$ reposi- 
ção de nicotina pela goma de mascar, associada à orientação comportamental, pode ser eficaz, além de uma estratégia de redução gradual do número de cigarros consumidos por dia.

\section{Gravidez}

A dependência de nicotina durante a gravidez é um fator de risco para a mãe e para o feto. Os efeitos adversos de fumar durante a gestação têm sido revisados em detalhe e incluem aborto espontâneo, nascimentos prematuros e recém-nascidos de baixo peso. A mortalidade desses bebês é maior, assim como a presença de problemas durante o desenvolvimento físico e psicológico, quando comparados aos filhos de não fumantes. ${ }^{151}$

Entre as gestantes, $30 \%$ são fumantes, sendo a maioria jovens entre 24 e 35 anos, com baixo nível de escolaridade, desempregadas ou subempregadas, segundo pesquisas epidemiológicas inglesas. ${ }^{152}$ Entre as gestantes orientadas nas consultas de pré-natal quanto aos benefícios obtidos com a interrupção do uso de tabaco para a própria pessoa e para o feto, apenas uma em cinco se propôs a interromper o uso. ${ }^{153}$ Os estudos que compararam grávidas aconselhadas com aquelas que não receberam aconselhamento não mostraram diferenças significativas na taxa de abstinência. ${ }^{154} \mathrm{Com}$ intervenções especiais, essas taxas aumentam em $10 \% .{ }^{155}$

O trabalho de motivação para permanecer em abstinência deve estender-se ao longo do período pós-parto, devido ao alto índice de recaída nessa fase. ${ }^{156} \mathrm{O}$ emprego da terapia de reposição de nicotina só está indicado quando os riscos de continuar fumando forem maiores que o risco da reposição.

\section{Fumantes hospitalizados}

O ambiente hospitalar não permite fumar e, além desse fato, a doença que causou a internação pode estar relacionada ao tabagismo. Por meio de orientação específica e da reposição de nicotina quando necessária, o paciente pode manter-se abstinente com menos sofrimento e, após a alta hospitalar, deverá ser encaminhado para tratamento especializado.

Uma internação parece ser um momento especial para se iniciar a motivação de um paciente para interromper o uso de tabaco, mas, em estudos clínicos ingleses, mesmo para aqueles pacientes com doenças relacionadas ao tabagismo, os resultados têm

\section{Referências}

1. Connolly G, Chen T. International health and tobacco use. In: Houston TP, ed. Tobacco use: an American crisis. Chicago: American Medical Association; 1993. p. 72.

2. Peto R, Lopez AD, Boreham J, Thun M, Heath C Jr. Mortality from smoking in developed countries 1950-2000. New York: Oxford University Press; 1994.

3. Centers for Disease Control and Prevention. Cigarette smokingatributable mortality and years of potential life lost, United States. MMWR 1990;42:645-9.

4. Centers for Disease Control and Prevention. Cigarette smoking and health among adults, United States, 1993. MMWR 1994;43:925-30. sido pouco efetivos, sendo a taxa de cessação do uso ainda menor quando comparada aos pacientes com outras doenças. ${ }^{75}$ Ao contrário, alguns pesquisadores obtiveram nesse ambiente um resultado mais adequado quanto à efetividade da intervenção com tratamentos especialmente desenhados para esses pacientes. ${ }^{98,157}$

\section{Ganho de peso}

Ansiedade em relação ao ganho de peso, principalmente entre as mulheres, é um fator importante que interfere no tratamento do tabagismo. ${ }^{158} \mathrm{~A}$ maioria dos fumantes vai ganhar $10 \%$ de seu peso em até cinco anos do início do tratamento. ${ }^{6}$ Cerca de $1 / 5$ dos fumantes ganha mais de $5 \mathrm{~kg} .{ }^{61}$ Mulheres e fumantes de mais de 25 cigarros ao dia apresentam maior risco para o ganho de peso. ${ }^{159}$

$\mathrm{O}$ ganho de peso parece ser devido a um aumento da ingesta e a um reajuste metabólico. O paciente deve ser alertado sobre a possibilidade de ganho de peso, porém deve-se enfatizar que a prioridade é deixar de fumar, e que o ganho de peso é limitado. Estimular o paciente a ingerir uma maior quantidade de frutas $\mathrm{e}$ verduras e a exercitar-se pode ajudar. A reposição de nicotina ou a bupropiona poderão ser prescritas com o objetivo de retardar o ganho de peso. ${ }^{102,160}$

\section{Considerações Finais}

A partir do que foi sintetizado neste consenso, recomenda-se que:

- todos os indivíduos devam ser abordados e interrogados sobre o uso do tabaco, principalmente os adolescentes;

- se fumantes, todos devem ser aconselhados, motivados e auxiliados a interromper o uso;

- o acesso ao tratamento deve ser facilitado em todos os níveis de atenção à saúde;

- a terapia de reposição da nicotina deve ser adotada para a maioria dos fumantes, exceto para grupos de risco como grávidas, idosos com doenças cardiovasculares e adolescentes. A terapia comportamental breve em grupo é um recurso importante para esse tratamento;

- novas medidas preventivas para a população geral devem ser utilizadas, pois o resultado esperado quanto à redução do fenômeno não tem sido efetivo.
5. American Thoracic Society. Cigarette smoking and health. Am J Resp Crit Care Med 1996;153:861-5.

6. US Department of Health and Human Services. The health consequences of smoking: nicotine addiction. A report of the Surgeon General. Public Health Service, Office on Smoking and Health. Rockville; 1988.

7. Fiore MC, Bailey WC, Cohen SJ. Smoking cessation. Clinical Practice Guideline, 18. Rockville (Md): US Department of Health and Human Services, Public Health Service, Agency for Health Care Policy and Research; 1996. [AHCPR Publication, 96-0692]. 
8. Fiore MC, Jorenby DE, Baker TB, Kenford SL. Tobacco dependence and the nicotine patch: clinical guidelines for effective use. JAMA 1992;268:2687-94.

9. Giovino GA, Henningfield JE, Tomar SL, Escobedo LG, Slade J. Epidemiology of tobacco use and dependence. Epidemiol Rev 1995;17(1):48-65.

10. Galduróz JCF, Noto AR, Nappo SA, Carlini EA. Primeiro levantamento domiciliar nacional sobre o uso de drogas psicotrópicas. Parte A: Estudo envolvendo as 24 maiores cidades do Estado de São Paulo. São Paulo: Unifesp/Cebrid; 1999.

11. Hirschman RS, Leventhal H, Glynn K. The development of smoking behavior: conceptualization and supportive cross-sectional survey data. J Appl Soc Psychol 1984;14(3):184-206.

12. McNeill A. The development of dependence on smoking in children. Br J Addict 1991;86:589-92.

13. US Department of Health and Human Services. Preventing tobacco use among young people: a report of the surgeon general. US Department Health and Human Services. Rockville: Public Health Service, Centers for Disease Control, National Center for Chronic Disease Prevention and Health Promotion, Office on Smoking and Health; 1994

14. Galduróz JCF; Noto AR, Carlini EA. IV Levantamento sobre o uso de drogas entre estudantes de $1^{\circ}$ e $2^{\circ}$ graus em 10 capitais brasileiras. São Paulo: UNIFESP/Cebrid; 1997.

15. American Psychiatric Association. Practice guideline for the treatment of patients with nicotine dependence. Am J Psychiatry 1996;151:1-31.

16. MacKenzie TD, Bartecchi CE, Schrier RW. The human costs of tobacco use (second of two parts). N Engl J Med 1994;330:975-80.

17. Owen L, Scott P. Barriers to good practice in smoking cessation work among pregnant women. J Inst Health Educ 1995;33:110-2.

18. Department of Health of London. Smoking kills. A white paper on tobacco. London: The Stationery Office; 1998.

19. Foulds J. Strategies for smoking cessation. In: Doll R, Crofton J, eds. Tobacco and Health. Br Med Bull 1996;52(1):157-73.

20. Henningfield JE, Keenan RM. Nicotine delivery kinetics and abuse liability. J Consult Clin Psychol 1993;61:743-50.

21. Benowitz NL. Nicotine safety and toxicity. New York: Oxford University Press; 1998.

22. Heishman SJ, Taylor RC, Henningfield JE. Nicotine and smoking: a review of effects on human performance. Exp Clin Psychopharmacol 1994;2:345-95.

23. Haxby DG. Treatment of nicotine dependence. Am Health Syst Pharmacol 1995;52:265-81.

24. Evans SM, Cone EJ, Henningfield JE. Rapid arterial kinetics of intravenous and smoked cocaine: relationship to subjective and cardiovascular effects. NIDA Research Monograph; 1995.

25. Gilbert DG, Wesler R. Emotion, anxiety and smoking. In: Ney T, Gale A, eds. Smoking and human behavior. Chichester: Wiley; 1989.

26. Meliska CJ, Gilbert DG. Hormonal and subjective effects of smoking the first five cigarettes of the day: a comparison in males and females. Pharmacol Biochem Behav 1991;40:229-35.

27. Clarke PBS. Recent progress in identifying nicotinic cholinoceptors in mammalian brain. Trends Pharmacol Sci 1987;8:32-5.

28. US Department of Health and Human Services. The health consequences of smoking: nicotine addiction. A report of the Surgeon General. Rockville: Public Health Service, Office on Smoking and Health; 1998.

29. Sargent PB. The diversity of neuronal nicotinic acetylcholine receptors. Ann Rev Neurosci 1993;16:403-43.

30. Leshner AI. Understanding drug addiction: implications for treatment. Hosp Pract 1996;47-59.

31. Nisell M, Nomikos GG, Svenson TH. Nicotine dependence, midbrain dopamine system and psychiatric disorders. Pharmacol Toxicol 1995;76:157-62.

32. Brodie MS. Low concentrations of nicotine increase the firing rate of neurons of the rat ventral tegmental area in vitro. In: Adlkofer F, Thurau K, eds. Effects of nicotine on biological systems. Birkhäuser: Basel; 1991. p. 373.
33. Wise RA, Hoffman DC. Localization of drug reward mechanisms by intracranial injections. Synapse 1992;10:247-63.

34. Clarke PBS, Pert A. Autoradiographic evidence for nicotine receptors on nigrostriatal and mesolimbic dopaminergic neurons. Brain Res 1985;348:355-8.

35. London ED. Effects of nicotine on cerebral metabolism. In: Boch G, Marsh J, eds. The biology of nicotine dependence. New York: Wiley; 1990. p. 131

36. Balfour DJ. The effects of nicotine on brain neurotransmitter systems. Pharmcol Ther 1982;16:269-82.

37. Mitchell SN. Role of the locus coeruleus in the noradrenergic response to a systemic administration of nicotine. Neuropharmacol 1993;32:937-49.

38. Richardson NR, Roberts DCS. Fluoxetine pretreatment reduces breaking points on a progressive ratio schedule reinforced by intravenous cocaine self-administration in the rat. Life Sci 1991;49:833-40.

39. Wada E, McKinnon D, Heinemann S, Patrick J, Swanson LW. The distribution of mRNA encoded by a new member of the neuronal nicotinic acetylcholine receptor gene family (a5) in the rat central neurons system. Brain Res 1990;526:45-53.

40. Fagerström KO, Schneider NG. Measuring nicotine dependence: a review of the fagerstrom tolerance questionnaire. J Behav Med 1989;12:159-82.

41. Araújo DM, Lapchak PA, Collier B, Quirion R. Characterization of $\mathrm{N}-(3 \mathrm{H})$ methylcarbamylcholine binding sites and effect of $\mathrm{N}$ methylcarbamylcholine on acetylcholine release in rat brain. $\mathrm{J}$ Neurochem 1988;51:292-9.

42. Clarke PBS. Nicotine and smoking: a perspective from animal studies. Psychopharmacol (Berl) 1987;92:135-43.

43. Benwell MEM, Balfour DJK, Khadra LF. Studies on the influence of nicotine infusions on mesolimbic dopamine and locomotor responses to nicotine. J Clin Invest 1994;72:233-9.

44. Corrigall WA, Franklin KBJ, Coen KM, Clarke PBS. The mesolimbic dopaminergic system is implicated in the reinforcing effects of nicotine. Psychopharmacol (Berl) 1992;107:285-9.

45. Boyle P. Cancer, cigarette smoking and premature death in Europe: a review icluding the recommendations of european cancer experts consensus meeting, Helsinki, October 1996. Lung Cancer 1997;17:1-60.

46. Henningfield JE, Keenan RM, Clarke PBS. Pharmacological aspects of drug dependence. In: Schuster CR, Kuhar MJ, eds. Springer, Toward an Integrated Neurobehavioral Approach. New York: Verlag Berlim Heidelberg; 1996. vol. 8. p. 271-314.

47. Kendler KS, Neale MC, Sullivan P, Corey LA, Gardner CO, Prescott CA. A population-based twin study in women of smoking initiation and nicotine dependence. Psychological Medicine. 1993;29(2):299308.

48. Foulds J. Detrimental effects of nicotine on mood? Addiction 1994;89:136-7.

49. Everett SA, Warren CW, Sharp D, Kann L, Husten CG, Crossett LS. Initiation of cigarette smoking and subsequent smoking behavior among U.S. high school students. Prev Med 1999;29(5):327-33.

50. Perkins KA. Metabolic effects of cigarette smoking. J Appl Physiol 1992;72:401-9.

51. Heishman SJ, Taylor RC, Henningfield JE. Nicotine and smoking: a review of effects on human performance. Exp Clin Psychopharmacol; 1994,2:245-395

52. Wennes K, Revell A, Warburton DM. Work and stress as motive for smoking. In: , Cumming G, Bonsignore G, eds. Smoking and the Lung. New York: Plenum; 1984. p. 233-48.

53. Henningfield JE, Cohen C, Slade JD. Is nicotine more addictive than cocaine? Br J Addict 1991;86:565-9.

54. Henningfield JE, London ED, Benowitz NL. Arterio-venous differences in plasma concentration of nicotine after cigarette smoking. JAMA 1990;263:2049-50.

55. West R, Russell M, Jarvis M, Pizzie T, Kadam B. Urinary adrenaline concentrations during 10 days of smoking abstinence. Psychopharmacol 1984;84:141-2. 
56. Heatherton TF, Kozlowski LT, Frecker RC, Fagerström KO. The fagerstrom test for nicotine dependence: a revision of the fagerstrom tolerance questionnaire. Br J Addict 1991;86:1119-27.

57. Hughes JR, Higgins ST, Hatsukami DK. Effects of abstinence from tobacco: a critical review. In: Kozlowski LT, Annis H, Cappel HD, Glaser F, Goodstadt M, Israel Y, Kalant H, Sellers EM, Vingilis J, eds. Research advances in alcohol and drug problems,10. New York: Plenum; 1990. p. 318.

58. Stolerman IP. Behavioural pharmacology of nicotine: multiple mechanisms. Br J Addict 1991;86:533-6.

59. DuPont RL, Gold MS. Withdrawal and reward: implications for detoxification and relapse prevention. Psychiatric Ann 1995;25:663-8.

60. Lando HA, Thai DT, Murray DM, Robinson LA, Jeffery RW, Sherwood NE, et al. Age of initiation, smoking patterns, and risk in a population of working adults. Prev Med 1999;29(6 Pt 1):590-8.

61. Hughes JR, Gulliver SB, Fenwick JW, Valliere WA, Cruser K, Pepper $\mathrm{S}$, et al. Smoking cessation among self-quitters. Health Psychol 1992;11:331-4.

62. Ferry LH, Grissino LM, Runfola PS. Tobacco dependence curricula in US undergraduate medical education. JAMA 1999;282(9):825-9.

63. US Department of Health and Human Services. Healthy People 2010 (Conference Edition in two volumes). Washington (DC); 2000.

64. Fiore MC, Jorenby DE, Schensky AE, Smith SS, Bauer RR, Baker TB. Smoking Status as the new vital sing: effect on assessment and intervention in patients who smoke. Mayo Clin Proc 1995;209-13.

65. Rechtine GR, Rechtine JC, Bolesta MJ. Smoking cessation in the spine surgeon's office: a review. J Spinal Dis 1999;12(6):477-81.

66. World Health Organization . International Statistical Classification of Diseases and related Health Problems, $10^{\text {th }}$ revision. Geneva: WHO, 1992.

67. American Psychiatric Association. Diagnostic and statistical manual of mental disorders. $4^{\text {th }}$ ed. Washington (DC): American Psychiatric Association; 1994.

68. Hurt RD. Treat tobacco dependence and "bend the trend". Bull World Health Org 1999;77(5):367.

69. Etter JF, Duc TV, Perneger TV. Validity of the Fagerstrom test for nicotine dependence and of the Heaviness of Smoking Index among relatively light smokers. Addict 1999;94(2):269-81.

70. Tiffany ST, Drobes DJ. Imagery and smoking urges: the manipulation of affective content. Addict Behav 1990;15:531-9.

71. Tiffany ST, Drobes DJ. The development and initial validation of a questionnaire of smoking urges. Br J Addict 1991;86:1467-76.

72. Hughes JR, Hatsukami DK. Sings and symptoms of tobacco withdrawal. Arch Gen Psychiatry 1986;43:289-94.

73. Kottke TE, Battista RN, DeFriese GH, Brekke ML. Attributes of successful smoking cessation interventions in medical practice: a meta-analysis of 39 controlled trials. JAMA 1988;259:28839.

74. Raw M, McNeill A, West R. Smoking Cessation Guidelines and their Cost Effectiveness. Thorax J Br Thorac Soc 1998;53(5):S1-S18.

75. Foulds J, Stapleton J, Hayward M, Russell MAH, Feyerabend C, Fleming $\mathrm{T}$, et al. Transdermal nicotine patches with low-intensity support to aid smoking cessation in outpatients in a general hospital. Arc Fam Med 1993;2:417-23.

76. Agency for Health Care Policy and Research. Clinical Practice Guideline. JAMA 1996;275(16):1270-80.

77. Addington J. Group treatment for smoking cessation among persons with schizophrenia. Psychiatric Serv 1998;49(7):925-8.

78. Benowitz NL. Nicotine addic. Primary care; Clin Off Prac 1999;26(3):611-31.

79. Covey LS. Tobacco cessation among patients with depression. Primary Care; Clin Off Prac 1999;26(3):691-70.

80. Mant D. R\&D in primary care. National working group report. Leeds: NHSE; 1997.

81. Pine D, Sullivan S, Conn SA, David C. Promoting tobacco cessation in primary care practice. Primary Care; Clin Off Prac 1999;26(3):591-610.

82. Perine JL, Schare ML. Effect of counselor and client education in nicotine addiction on smoking in substance abusers. Addict Behav 1999;24(3):443-7.
83. Severson HH, Hatsukami D. Smokeless tobacco cessation. Primary Care; Clin Off Prac 1999;26(3):529-51.

84. Fagerström KO, Tejding R, Westin A, Lunell E. Aiding reduction of smoking with nicotine replacement mediations: hope for the recalcitrant smoker? Tobacco Control 1997;6(4):311-6.

85. Hatsukami D, Jensen J, Allen S, Grillo M, Bliss R. Effects of behavioral and pharmacological treatment on smokeless tobacco users. J Consult Clin Psychol 1996;64(1):153-61.

86. Parrott S, Godfrey C, Raw M, West R, McNeill A. Guidance for commissioners on the cost effectiveness of smoking cessation interventions. Health Educ Authority Thorax 1998;53(5):S1-38.

87. Bandura A. Self-efficacy: Towards a unifying theory of behavioral change. Psychol Rev 1977;84:199-215.

88. Benowitz NL. Nicotine replacement therapy: What has been accomplished-can we do better? Drugs 1993;45:157-70.

89 . West $\mathrm{R}$. The nicotine replacement paradox in smoking cessation: how does nicotine gum really work? Br J Addict 1992;87:165-7.

90. Silagy C, Ketteridge S. Physician advice for smoking cessation (Cochrane review). In: The Cochrane Library, Issue 2. Oxford: Update Software; 1999.

91. Jorenby DE, Leischow SJ, Nides MA, Rennard SI, Johnston JA, Hughes AR, et al. A controlled trial of sustained-release bupropion, a nicotine patch, or both for smoking cessation. N Engl J Med 1999;340(9):685-91.

92. Stapleton J, Russell M, Feyrabend C, Wiseman S, et al. Dose effects and predictors of outcome in a randomized trial of transdermal nicotine patches in general practice. Addict 1995;90:31-42.

93. Hughes JR, Goldstein MG, Hurt RD, Shiffman S. Recent advances in the pharmacotherapy of smoking. JAMA 1999;281(1):72-6.

94. Jorenby DE, Hatsukami DK, Smith SS, Fiore MC, Allen S, Jensen J, et al. Characterization of tobacco withdrawal symptoms: transdermal nicotine reduces hunger and weight gain. Psychopharmacol 1996;128(2):130-8.

95. Alterman AI,Gariti P, Cook TG, Cnaan A. Nicodermal patch adherence and its correlates. Drug Alcohol Depend 1999;53(2):159-65.

96. Shaw JP, Ferry DG, Pethica D, Brenner D, Tucker IG. Usage patterns of transdermal nicotine when purchased as a non-prescription medicine from pharmacies. Tobacco Control 1998;7(2):161-7.

97. Shiffman S, Gitchell J, Pinney JM, Burton SL, Kemper KE, Lara EA. Public health benefit of over-the-counter nicotine medications. Tobacco Control 1997;6(4):306-10.

98. Rigotti NA, Arnsten JH, McKool KM, Wood-Reid KM, Singer DE, Pasternak RC. The use of nicotine-replacement therapy by hospitalized smokers. Am J Prev Med 1999;17(4):255-9.

99. Roussel G, Roche D, Momas I, Brahimi N, Callais F, Lequang NT, et al. Usefulness of markers in managing tobacco withdrawal. Pathol Biol 1997;45(6):467-71.

100.Fagerström KO. Combined use of nicotine replacement products. Health Values 1994;18(3):15-20.

101. Danis PG, Seaton TL. Helping your patients to quit smoking. Am Family Physician 1997;55(4):1207-14.

102. Dale L, Hurt R, Offord K, Lawson G. High dose nicotine patch therapy percentage of replacement and smoking cessation. JAMA 1995;274:1353-2.

103. Hughes JR, Gust SW, Skoog K, Keenan RM, Fenwick JW. Symptoms of tobacco withdrawal. A replication and extension. Arch Gen Psychiatry 1991;48:52-9.

104. Fagerström KO. Effectiveness of nicotine pach and nicotine gum as individual versus combined treatments for tobacco withdrawal symptoms.Psychopharmacol 1993;111:271-7.

105. Silagy C, Mant D, Fowler G, Lodge M. Meta-analysis on efficacy of nicotine replacement therapies in smoking cessation. Lancet 1994;343:139-42.

106. Henningfield JE. Do nicotine replacement medications work? A unique standard for nicotine. Addict 1994;89:434-6.

107. Patten CA, Rummans TA, Croghan IT, Hays JT. Development of depression during placebo-controlled trials of bupropion for smoking cessation: case reports. J Clin Psychiatry 1999;60(7):436-41.

108. Goldstein MG. Bupropion sustained release and smoking cessation. J Clin Psychiatry 1998;59(4):66-72. 
109. Deagle EA $3^{\text {rd }}$, Berigan TR. Adding behavioral therapy to medication for smoking cessation. JAMA 1999;281(21):1983-5.

110. Shiffman S, Johnston JA, Khayrallah M, Elash CA, Gwaltney CJ, Paty JA, et al. The effect of bupropion on nicotine craving and withdrawal. Psychopharmacol 2000;148(1):33-40.

111. Hurt RD, Sachs DP, Glover ED, Offord KP, Johnston JA, et al. Bupropion for smoking cessation: a double-blind, placebo-controlled dose response trial. N Engl J Med 1997;337:1195-202.

112. Prochazka AV, Weaver MJ, Keller RT, Fryer GE, Licari PA, Lofaso D. A randomized trial of nortriptyline for smoking cessation. Arch Internal Med 1998;158(18):2035-9.

113. Glassman AH. Cigarette smoking: implications for psychiatric illness. Am J Psychiatry 1993;150(4):546-53.

114. Gourlay SG, Benowitz NL. Is clonidine an effective smoking cessation therapy? Drugs 1995;50:197-207.

115. Jarvik ME, Henningfield JE. Pharmacological adjuncts for the treatment of tobacco dependence. In: Orleans CT, Slade J, eds. Nicotine addiction: principles and management. New York: Oxford University Press, 1993.

116. Hilleman DE, Mohiuddin SM, Del Core MG, Sketch MH. Effect of buspirone on withdrawal symptoms associated with smoking cessation. Arch Intern Med 1992;52:350-2.

117. Christen AG. Helping patients quit smoking: Lessons Learned in the trenches. Quintessence Int 1998;29(4):253-9.

118. Cinciripini PM, Cinciripini LG, Wallfisch A, Haque W, Van Vunakis H. Behavior therapy and the transdermal nicotine patch: effects on cessation outcome, affect, and coping. J Consult Clin Psychol 1996;64(2):314-23.

119. Cincipirini PM, Wetter DW, McClure JB. Scheduled reduced smoking: effects on smoking abstinence and potential mechanisms of action. Addict Behav 1997;22(6):759-67.

120. Hugues JR, Liguori A. Bibliographical analysis of research on smoking cessation therapy. Tobacco Control 1997;6(2):111.

121. Lichtenstein E, Hollis J. Patient referral to a smoking cessation program: who follows through? J Fam Pract 1992;34(6):739-44.

122. Reid RD, Pipe A, Dafoe WA. Is telephone counselling a useful addiction to physician advice and nicotine replacement therapy in helping patients to stop smoking? A randomized controlled trial. CMAJ 1999;160(11):1577-81.

123. Abbot NC, Stead LF, White AR, Barnes J, Ernst E. Hypnotherapy for smoking cessation (Cochrane review). In: The Cochrane Library, Issue 2. Oxford: Update Software; 1999.

124. Colsher PL, Wallace RB, Loeffelholz PL, Sales M. Health status of older male prisoners: a comprehensive survey. Am J Pub Health 1992;82:881-4.

125. Foulds J. The relationship between tobacco use and mental disorders. Curr Opin Psychiatry 1999;12:303-6.

126. Ellingstad TP, Sobell LC, Sobell MB, Cleland PA, Agrawal S. Alcohol abusers who want to quit smoking: implications for clinical treatment. Drug Alcohol Depend 1999;54(3):259-65.

127. Cinciripini PM, Hecht SS, Henningfield JE, Manley MW, Kramer BS. Tobacco addiction: implications for treatment and cancer prevention. J Natl Cancer Inst 1997;89(24):1852-67.

128. US Department of Health and Human Services. The health benefits of smoking cessation: a report of the Surgeon-General. Rockville (MD): USDHHS; 1990. [US DHHS Publication, (CDC) 90-8416].

129. Gritz ER. Smoking and smoking cessation in cancer patients. Br J Addict 1991;86:549-54.

130. Wald NJ, Hackshaw AK. Cigarette smoking: an epidemiological overview. Br Med Bull 1996;52:3-11.

131. Ostroff JS, Jacobsen PB, Moadel AB, Spiro RH. Prevalence and predictors of continued tobacco use after treatment of patients with head and neck cancer. Cancer 1995;75:569-76.

132. Instituto Nacional do Câncer. Falando sobre tabagismo. Coordenação Nacional de Controle do Tabagismo e Prevenção Primária. Ministério da Saúde, CONTAPP; 1996.

133. Iribarren C, Tekawa IS, Sidney S, Friedman GD. Effect of cigar smoking on the risk of cardiovascular disease, chronic obstructive pulmonary disease, and cancer in men. $\mathrm{N}$ Engl $\mathrm{J}$ Med 1999;340:1773-80.
134. Rogers WJ, Coggin CJ, Gersh BJ, Firsher LD, et al. Ten year followup of quality of life in patients randomized to receive medical therapy or coronary bypass graft surgery. The Coronary Artery Surgery Study (CASS). Circ 1990;82:1647-58.

135. Cupples ME, McNight A. Randomized controlled trial of health promotion in general practice for patients at higth cardiovascular risk. Br Med J 1994;309:993-6.

136. Jolly K, Bradley F, Sharp S, Smith H. Randomized controlled trial of follow-up care in general practice of patients with myocardial infarction and angina: final results of the Southampton Heart Integrated Care Project (SHIP). Br Med J 1999;318:706-11.

137. British Thoracic Society. Comparison of four methods of smoking withdrawal in patients with smoking related disease. Br Med J 1983;286:595-7.

138. British Thoracic Society. Smoking withdrawal in hospital patients: factors associated with outcome. Thorax 1984;39:651-6.

139. British Thoracic Society. Smoking cessation in patients: two further studies by the British Thoracic society. Thorax 1990;45:835-40.

140. Vineis P, Caporaso N. Tobacco and Câncer: epidemiology and the Laboratory. Environ Health Perspect 1995;103:156-60.

141. Thomas M, Walker A, Wilmot A, Bennett N. Living in Britain: results from the 1996 general household survey. Office for National Statistics. London: The Stationery Office; 1998.

142. Orleans CT, Resch N, Noll E, Keintz MK, Rinner BK, Brown TV. Use of transdermal nicotine in a state-level prescription plan for the elderly. A first look at 'real-word' patch users. JAMA 1994;271(8):601-7.

143. Barton J. Young teenagers and smoking in 1997. A report of the key findings from the teenage smoking attitudes survey carried out in England in 1997. London: Office for National Statistics; 1998.

144. Lynch BS, Bonnie RJ. Growing up tobacco free: preventing nicotine addiction in children and youths. Institute of Medicine. Washington (DC): National Academy Press; 1994

145. Goddard E. Why children start smoking. London: HMSO; 1990.

146. Sussman S, Lichtman K, Ritt A, Pallonen TE. Effects of thirtyfour adolescent tobacco use cessation and prevention trials on regular users of tobacco products. Subst Use Misuse 1999;34(11):1469-503.

147. Lamkin LP, Houston TP. Nicotine dependency and adolescents: preventing and treating. Primary Care; Clin Off Prac 1998;25(1):123-35.

148. Stanton RW. DSM-III-R tobacco dependence and quitting during late adolescence. Addict Behav 1995;20:595-603.

149. Sargent JD, Mott LA, Stevens M. Predictors of smoking cessation in adolescents. Arch Pediatr Adolesc Med 1998;152:388-93.

150. Najman JM, Lanyon A, Andersen M, Williams G, Bor W, O'Callaghan $\mathrm{M}$. Socioeconomic status and maternal cigarette smoking before, during and after a pregnancy. Aust N Zealand J Public Health 1998;22(1):60-6.

151. Charlton A. A children and smoking: the family circle. Br Med Bull 1996;52:99-107.

152. Walsh RA, Redman S, Brinsmead MW, Byrne JM, Melmeth A. A smoking cessation program at a public antenatal clinic. Am J Public Health 1997;87(7):1201-4.

153. Owen L, McNeil A, Callum C. Trends in smoking during pregnancy in England, 1992-7: quota sampling surveys. Br Med J 1998;317:728.

154. Wisborg K, Henriksen TB, Secher NJ. A prospective intervention study of stopping smoking in pregnancy in a routine antenatal care setting. Br J Obstetr Gynaecol 1998;105:1171-6.

155. West R, Gossop MA. A comparison of withdrawal symptoms from different drug classes. Addict 1994;89:1483-9.

156. Manfredi C, Crittenden KS, Warnecke R, Engler J, Cho YI, Shaligram C. Evaluation of a motivational smoking cessation intervention for women in public health clinics. Prev Med 1999;28(1):51-60.

157. Miller NH, Smith PM, DeBusk RF, Sobel DS, Taylor CB. Smoking cessation in hospitalized patients. Results of a randomized trial. Arch Internal Med 1997;157(4):409-15. 
158. Marcus BH, Albrecht AE, King TK, Parisi AF, Pinto BM, Roberts M, et al. The efficacy of exercise as an aid for smoking cessation in women: a randomized controlled trial. Arch Internal Med 1999;159(11):1229-34.

159. O'Hara P, Connett JE, Lee WW, Nides M, Murray R, Wise R. Early and late weight gain following smoking cessation in the Lung health study. Am J Epidemiol 1998;148(9):821-30.

160. Hurt RD, Fant RV, Tomar SL. Smokeless tobacco: an addicting drug. Advanc Dental Res 1997;11(3):330-5.
Correspondência

Ana Cecilia Petta Roselli Marques

Depto de Psicobiologia da Universidade Federal de São Paulo

Rua Napoleão de Barros, 925 , Vila Clementino

4024-002 São Paulo, SP, Brasil

Tel.: (0xx11) 5539-0155

E-mail: anace@psicobio.epm.br

\section{Anexo 2}

\section{Questionário de Tolerância de Fagerström}

Tabagista?

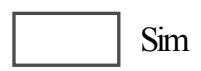
Não

1- Quanto tempo depois de acordar, você fuma o seu primeiro cigarro?

$\begin{array}{ll}0 & \text { Após } 60 \text { minutos } \\ 1 & 31-60 \text { minutos } \\ 2 & 6-30 \text { minutos } \\ 3 & \text { Nos primeiros } 5 \text { minutos }\end{array}$

2- Você encontra dificuldades em evitar fumar em lugares onde é proibido, como por exemplo: igrejas, local de trabalho, cinemas, shoppings etc.?

$\begin{array}{ll}0 & \text { Não } \\ 1 & \text { Sim }\end{array}$

3- Qual é o cigarro mais difícil de largar ou de não fumar?

$\begin{array}{ll}0 & \text { Qualquer um } \\ 1 & \text { O primeiro da manhã }\end{array}$

4- Quantos cigarros você fuma por dia?

$\begin{array}{ll}0 & 10 \text { ou menos } \\ 1 & 11 \text { a } 20 \\ 2 & 21 \text { a } 30 \\ 3 & 31 \text { ou mais }\end{array}$

5- Você fuma mais freqüentemente nas primeiras horas do dia do que durante o resto do dia?

$\begin{array}{ll}0 & \text { Não } \\ 1 & \text { Sim }\end{array}$

6- Você fuma mesmo estando doente ao ponto de ficar acamado a maior parte do dia?

$\begin{array}{ll}0 & \text { Não } \\ 1 & \text { Sim }\end{array}$

\begin{tabular}{|c|c|c|}
\hline Pontuação: & Leve & 0 a 4 \\
\hline 2 & Médio & 5 a 7 \\
\hline 3 & Alto & 8 a 11 \\
\hline
\end{tabular}


Anexo 1- Ficha do paciente

Identificação:

Nome:

Idade:

Sexo:

Dados vitais (de acordo com a especialidade do profissional):

Peso:

Pulso:

PA:

FC:

FR:

Uso de tabaco:

Nunca

Eventual; quanto

Freqüente; quanto

Já fez tratamento anterior:

Quantos

Ficou abstinente por quanto tempo
Anexo 4 - Recomendação para a elaboração de material de auto-ajuda

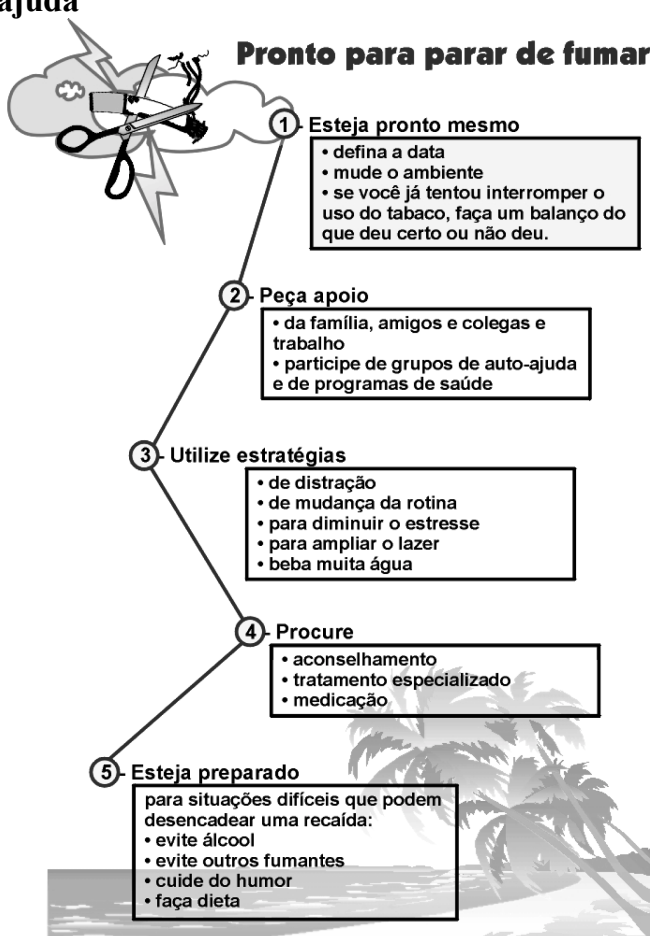

Anexo 3

\section{Estrutura do aconselhamento}

Apesar de todas recomendações serem centradas na relação médico-paciente, toda equipe deve ser envolvida.

$1^{\circ}$ PASSO - Identifique e documente sistematicamente todos os fumantes em todas as consultas. Isto pode ser feito incluindo perguntas sobre o hábito de fumar quando é feita sua identificação em qualquer nível de atenção à saúde.

$\mathrm{O}(\mathrm{A})$ sr./sra. já tentou parar de fumar? $\mathrm{O}(\mathrm{A})$ sr./sra. teria interesse em tentar?

$2^{\circ}$ PASSO - Aconselhe todos os fumantes a parar. $\mathrm{O}$ aconselhamento deve ser claro e objetivo. Intervenções motivacionais adequadas podem facilitar esse processo. Cada paciente deve compreender a relevância que o hábito de fumar tem no seu caso; receber informação personalizada sobre os riscos agudos e em longo prazo se continuar fumando e ser convidado a pensar sobre os benefícios em parar de fumar. Essas intervenções devem ser repetidas em cada consulta.

$3^{\circ}$ PASSO - Ajude o paciente a parar. Se o paciente mostra-se interessado em parar de fumar, o profissional deve ajudá-lo a estruturar um plano: estabeleça uma data para a parada. Idealmente, dentro de 15 dias; revise experiências anteriores como o que o ajudou a parar; o que causou problemas; a intensidade da síndrome de abstinência e como a enfrentou, e que fatores estavam implicados na recaída. Prepare o ambiente, eliminando cigarros e objetos relacionados de seu ambiente doméstico, trabalho, carro etc. Informe familiares, amigos e colegas de trabalho sobre a tentativa de parar. Solicite seu apoio e compreensão. Pense como irá proceder em relação ao assunto de bebidas alcoólicas, pois seu consumo está diretamente relacionado ao fumar e à possibilidade de recaídas. Antecipe as prováveis situações de risco desafiadoras, planejando modos de enfrentamento. Use alguma forma de reposição de nicotina, como goma de mascar e adesivos sob orientação. O uso desses produtos pode duplicar as chances de sucesso, diminuindo os sintomas de abstinência da nicotina e a vontade intensa de fumar. Material educativo ilustrado adequado pode auxiliar no tratamento.

$4^{\circ}$ PASSO - A primeira consulta de acompanhamento deve ser marcada no máximo até 7 dias após a data da parada, para melhor manejo dos sintomas de abstinência e das compulsões. A consulta seguinte não deve passar de 30 dias e, se necessário, mais vezes dentro dos seis meses de abstinência. Muitas vezes são necessárias várias tentativas até que se consiga parar com sucesso. Quando o fumante fracassa após repetidas tentativas de parar ou tem sintomas graves de abstinência, deve ser encaminhado para um serviço de tratamento especializado.

$5^{\circ}$ PASSO - Planejar o seguimento para o período após a alta com o objetivo de manutenção de abstinência por pelo menos mais quatro sessões. 